\title{
THE FUTURE OF TELECOMMUNICATIONS SYSTEMS AS APPLIED TO METEOROLOGY
}

\author{
Harold Gruen \\ Philco-Ford Corporation, Willow Grove, Pa.
}

\begin{abstract}
Summary
Improvements in telecommunications during the next decade will markedly improve both the amount and reliability of meteorological data. Synoptic data will be available in almost real time on a global basis.

While this facility will become more available during the next decade, it is not without problems. While the amount of available telecommunications is rapidly increasing, it is tempered by potential problems. Information bandwith and transmission time are directly related to power and cost. These, in turn, contrast with precision, accuracy and quantity of data required.

Obviously, the greatest degree of flexibility in the kind, amount and quality of data leads to the greatest complexity. Trade-offs among several parameters must be considered.

The key ingredient to the communications is the satellite. By placing an electronic platform many thousands of miles above the earth, many meteoro-
\end{abstract}

logically interesting things are possible:

1) A microscopic view of the earth can be taken. Large-scale trends and interactions can be observed. Improvements in sensors and electronic data gathering further enable greater precision and hence improved quality data.

2) Given a relatively stable platform that can "see" large expanses of earth simultaneously, synoptic data gathering is feasible and practical. As the satellites improve in performance, the communication terminal associated with the sensor becomes less complex and expensive.

3) Real-time communications on a global scale are achievable.

Standardization of sensors and communication channels will have to be developed. Man and computer conversational modes, too, will have to be developed. Meteorologists will have to understand these technologies to develop a best set of standards that has a maximum effectiveness relating to their particular needs. 